\title{
Emitter-Metasurface Interface for Manipulating Emission Characteristics of Quantum Defects
}

\author{
Pankaj K. Jha ${ }^{1,}$, , Ghazaleh K. Shirmanesh ${ }^{1}$, Hamidreza Akbari ${ }^{1}$, Meir Y. Grajower ${ }^{1}$, Claudio G. Parazzoli ${ }^{2}$, \\ Benjamin E. C. Koltenbah ${ }^{3}$, Harry A. Atwater ${ }^{1}$ \\ ${ }^{I}$ Thomas J. Watson Laboratory of Applied Physics and Materials Science, California Institute of Technology, Pasadena CA 91125 USA \\ ${ }^{2}$ North West Quantum Science, Seattle WA 98198 USA \\ ${ }^{3}$ The Boeing Company, P. O. Box 3703 Seattle WA 98124 USA \\ *Author e-mail address: pkjha@caltech.edu
}

Abstract: We demonstrate a chip-scale quantum emitter-metamaterial device that emits highly directional photons. Our device opens the door for quantum imaging of weak sources by adding photon(s) to manipulate the photon statistics for improved signal-to-noise ratio. (ㅇ 2020 The Author(s)

\section{Introduction}

Single-photon sources are elementary building blocks for photonic quantum networks, quantum information processing, and quantum metrology, where the photons are used as "flying" qubits [1]. Several solid-state sources [2] like single organic molecules, self-assembled quantum dots, and semiconductor nanocrystals have been presented as potential emitter candidates. However, the ideal emitter candidate should be photostable, work at room temperature, and easy to control and manipulate. Recent discovery of quantum light emission from twodimensional (2D) van der Waals (vdW) materials, such as transition metal dichalcogenides (TMDCs) [3] and hexagonal boron nitride $(h \mathrm{BN})$ [4] have opened the door to a new class of single-photon sources. In contrast to other solid-state emitter candidates, these emitters have much narrower emission spectra at room temperature and they can be controlled electrically. Moreover, 2D materials may facilitate easier integration into complex photonic structures for onchip quantum applications.

Here, we report a chip-scale optical interface configuration for coupling $h \mathrm{BN}$ color-centers with planar arrays of classical nanoantennas, configured as a metasurface $[5,6]$. A schematic representation of our $h \mathrm{BN}$-metasurface interface is shown in Fig. 1. Here, an atomically thin layer of $h \mathrm{BN}$ is transferred onto a phase gradient metasurface. This hybrid device, when illuminated by 532

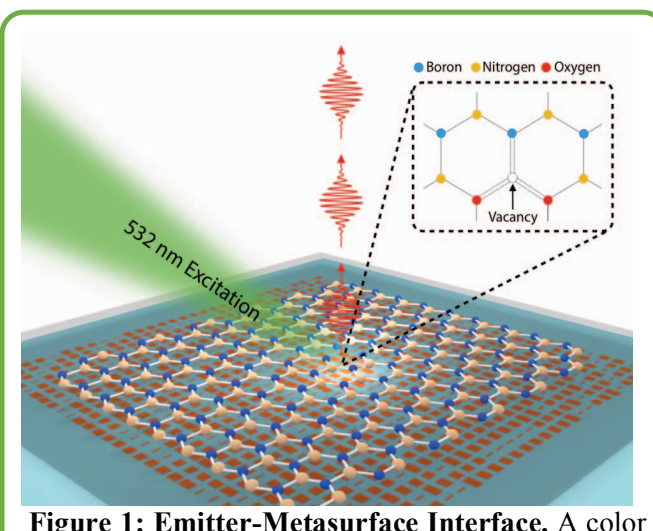

Figure 1: Emitter-Metasurface Interface. A color center in hexagonal boron nitride in the vicinity of a phase gradient metasurface. One such color centers are shown in the inset where two oxygen atom have replaced two boron atoms and vacancy at the site of a nitrogen atom. $\mathrm{nm}$ laser, will emit single-photons from a color-center in $h \mathrm{BN}$ along the surface-normal direction, thus creating highly directional single-photon source.

\subsection{Spectroscopy of color-centers in exfoliated $h B N$}

In our experiments, we exfoliated few-layer $h \mathrm{BN}$ samples on silicon $\left(\mathrm{Si} / \mathrm{SiO}_{2}\right)$ substrates. The sample $\left(h \mathrm{BN}-\mathrm{Si} / \mathrm{SiO}_{2}\right)$ was annealed in an inert environment $\left(\mathrm{Ar}-\mathrm{N}_{2}\right)$ at 850 Celsius and we performed optical characterization using a
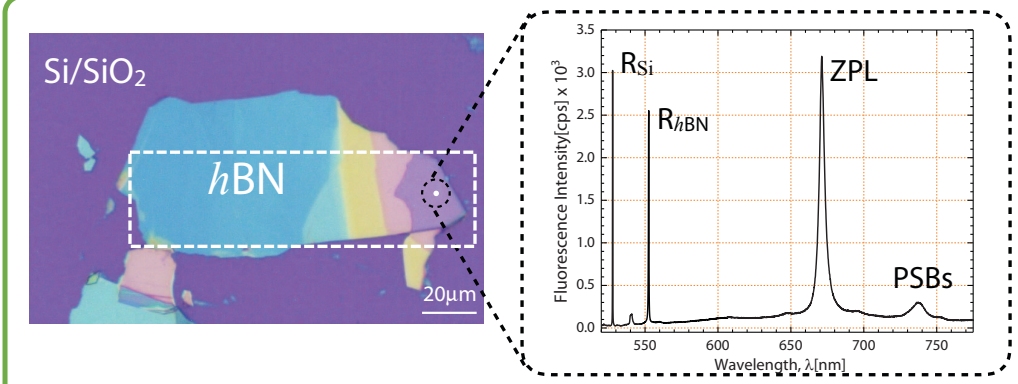

Figure 2: Photoluminescence spectrum from one of the color centers in an exfoliated $h \mathrm{BN}$ flake on silicon substrate which was annealed at 850 degrees Celsius in an inert environment. confocal Raman microscope. Figure 2 (a) shows one of the exfoliated flakes. Fig. 2(b) illustrates the photoluminescence (PL) spectrum from a location highlighted by the black circle indicated on the image. We observed a sharp emission line characteristic of zero-phonon line (ZPL) in $h \mathrm{BN}$ at $\sim 665 \mathrm{~nm}$. The fullwidth at half-maximum (FWHM) of the $\mathrm{ZPL}$ is $\sim 2-3 \mathrm{~nm}$ at room temperature with characteristic vibronic sidebands redshifted by $\sim 160 \mathrm{meV}$. We also performed PL mapping of the flake in 
the region shown by the white dashed line and we found several color centers with characteristic ZPL in the range $600-700 \mathrm{~nm}$ with photon counts as high as several thousand per second.

\subsection{Design and fabrication of a phase-gradient metasurface}

We designed a phase gradient metasurface configured so that emission from a dipole located at $(0,0, z)$ is highly directional (as shown in Fig. 3 left panel). We used six different silver bar nanoantenna elements to realsize a

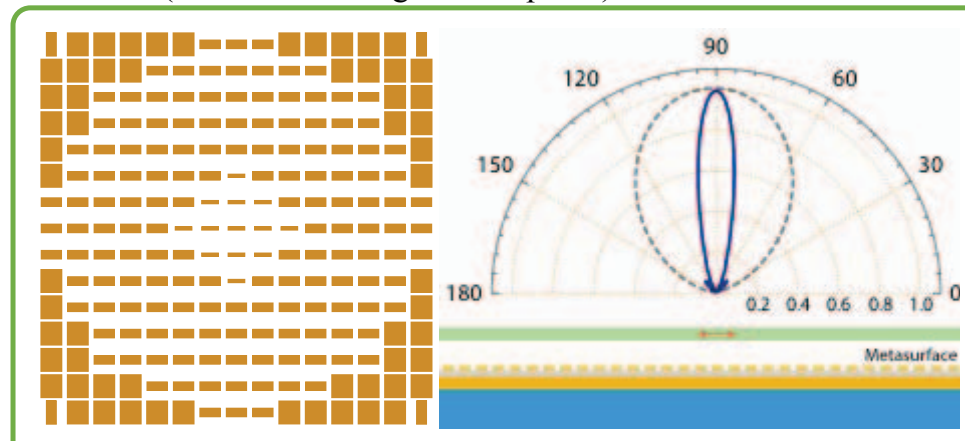

Figure 3: Metasurface design and far-field radiation pattern of an atomic dipole located at the focal spot of the metasurface. metasurface whose phase gradient ranges from $0-2 \pi$ with a step size of $\pi / 6$. The thickness of the nanoantennas is $30 \mathrm{~nm}$ and the thickness of the dielectric spacer layer (aluminum dioxide) is $65 \mathrm{~nm}$. The phase shift and reflection efficiency is calculated using a commercial full-wavelength simulation package (COMSOL Multiphysics). The average reflection intensity of the nanoantennas was $\sim 81 \%$. In Fig. 3, we have plotted far-field radiation pattern of an atomic-dipole with dipole orientation along the surface of the metasurface. Our simulation shows a highly directional (surface-normal) emission from the emitter (solid blue). The dashed curve shows the radiation pattern without the metasurface.

To summarize, we have introduced and theoretically demonstrated a chip-scale interface between a defect-based quantum emitter and a phase gradient metasurface. We show that a judiciously designed metasurface can engineer far-field radiation pattern of an emitter located at its focal spot such that the emission is highly directional. Our device also opens the door a similar system that favorably modifies photon statistics of a weak input thermal beam, increases photon count leading to larger signal-to-noise ratio and enhanced images of the source [7].

\section{References:}

(1) J. P. Dowling and G. J. Milburn, "Quantum technology: the second quantum revolution," Phil. Trans. R. Soc. Lond. A 361, 1655 (2003).

(2) W. E. Moerner, "Single-photon sources based on single molecules in solids," New J. Phys. 6, 88 (2004).

(3) A. Srivastava, M. Sidler, A. V. Allain, et al., "Optically active quantum dots in monolayer WSe, ," Nat. Nanotechnol. 10, 491 (2015).

(4) T. T. Tran, K. Bray, M. J. Ford, et al., "Quantum emission from hexagonal boron nitride monolayers," Nat. Nanotechnol. 11, 37 (2016).

(5) P. K. Jha, X. Ni, C. Wu, et al., "Metasurface-Enabled Remote Quantum Interference," Phys. Rev. Lett. 115, 025501 (2015).

(6) P. K. Jha, N. Shitrit, X. Ren, et al., "Spontaneous Exciton Valley Coherence in Transition Metal Dichalcogenide Monolayers Interfaced with an Anisotropic Metasurface," Phys. Rev. Lett. 121, 116102 (2018).

(7) C. G. Parazzoli, B. E. Koltenbah, D. R. Gerwe, et. al., "Enhanced Thermal Objective Imaging by Photon Addition or Subtraction", arXiv 160902780(2016). 